J. Clin. Chem. Clin. Biochem.

Vol. 22, 1984, pp. 385-388

\title{
Einfache und rasche Bestimmung von Thiopental im Serum mit HPLC
}

\author{
Von H. Gruhl und H. Mayer \\ Institut für Laboratoriumsmedizin, Zentralklinikum Augsburg
}

(Eingegangen am 20. Dezember 1983/14. Februar 1984)

Zusammenfassung: Es wird eine schnelle, präzise und empfindliche Methode zur Bestimmung von Thiopental im Serum mittels HPLC beschrieben. Die Analyse dauert einschließlich Probenvorbereitung 20 Minuten. Die Präzision in der Serie beträgt 1,5\%, von Tag zu Tag 3,8\%. Die Nachweisgrenze liegt bei $380 \mathrm{nmol} / \mathrm{l}$.

\section{A simple and rapid determination of serum thiopental by HPLC}

Summary: An HPLC method is described for the determination of thiopental in serum. It offers the advantage of a simple extraction procedure and a low detection limit. Including sample preparation, the analysis takes 20 minutes. The within-run and between-run CV's were $1.5 \%$ and $3.8 \%$ respectively.

\section{Einführung}

Neben der Verwendung als Kurznarkotikum findet Thiopental in zunehmendem Maße Eingang in die „protektive“ Therapie bei Patienten mit SchädelHirn-Traumen und Hirnödemen, da Thiopental in hoher Dosierung appliziert den intrakraniellen Druck deutlich zu senken vermag $(1,2)$. Die hohe Dosierung sowie die besōndere Pharmakokinetik von Thiopental machen eine engmaschige, aktuelle Kontrolle der Thiopentalkonzentration im Serum erforderlich.

Thiopentalbestimmungen wurden bisher entweder photometrisch (3), mittels GC (4-6) oder HPLC $(7-10)$ durchgeführt. Die photometrischen Analysen sollten wegen der Unspezifität verlassen werden. Die gaschromatographischen Methoden und ebenso die bịsher veröffentlichten Verfahren in der HPLC benötigen eine umfangreiche Probenvorbereitung; damit ist eine rasche Kontrolle der Thiopentalkonzentration nicht gegeben. Wir haben deshalb eine Methode zur genauen quantitativen Bestimmung von Thiopental mittels HPLC entwickelt, die sich durch einfachste Probenvorbereitung, schnelle Analysenzeit sowie hohe Empfindlichkeit und Reproduzierbarkeit auszeichnet.

\section{Material und Methoden}

Die Analysen wurden mit einem Flüssigkeitschromatographen der Firma Hewlett Packard (Typ 1084 B) mit variablem Wellenlängendetektor (Typ HP $79875 \mathrm{~A}$ ) durchgeführt.

Methanol und Ethylacetat (Lichrosolv-Qualität) wurden von der Firma Merck, Darmstadt, bezogen. Das verwendete bidestillierte Wasser stammte aus eigener Produktion.

\section{Kalibrierstandards und Patientenproben}

Na-Thiopental wurde als Trockensubstanz einer Ampulle Trapa$\mathrm{nal}^{\otimes}$ (Fa. Byk-Gulden) entnommen. Kalibrierstandards wurden durch Aufstocken von thiopentalfreiem gepooltem Serum mit NaThiopental hergestellt. Wegen der Linearität und der guten Reproduzierbarkeit der Kalibriergeraden genügt das Mitführen eines einzigen Standards $(20 \mathrm{mg} / \mathrm{l}$ entsprechend $75,7 \mu \mathrm{mol} / / \mathrm{Na}$ Thiopental).

\section{Extraktion von Thiopental aus Serum}

$100 \mu \mathrm{l}$ der Standard- bzw. Patientenseren wurden mit 100 $\mu \mathrm{l}$ Ethylacetat versetzt, das Gemisch $15 \mathrm{~s}$ lang mit einem Whirlmixer geschüttelt und danach für $3 \mathrm{~min}$ bei $6000 \mathrm{~g}$ in einer Tischzentrifuge (Fa. Eppendorf, Typ 5414) zentrifugiert. Die überstehende thiopentalhaltige Ethylacetatphase wurde direkt in den Flüssigkeitschromatographen injiziert.

\section{Chromatographisches System}

Zur Chromatographie wurde eine 10 $\mu \mathrm{m}$ RP-8 Säule von Hewlett Packard, Länge $250 \mathrm{~mm}$, innerer Durchmesser $4,6 \mathrm{~mm}$, eingesetzt. Die Säule wurde auf $35^{\circ} \mathrm{C}$ temperiert. Das injizierte Pro- 
benvolumen betrug bei allen Experimenten $10 \mu$ l. Eluiert wurde mit einem Wasser-Methanol Gemisch mit einem Methanolanteil von 0.55 für $6 \mathrm{~min}$. Anschließend wurde die Säule mit Methanol (Volumenanteil 0,95) gespült und $3 \mathrm{~min}$ mit wäßr. Methanol (Volumenanteil 0,55) rekonditioniert. Die Flußrate betrug $2 \mathrm{ml} / \mathrm{min}$. Die Absorption wurde bei $290 \mathrm{~nm}$ (Referenzwellenlänge $460 \mathrm{~nm}$ ) registriert; die Auswertung erfolgte über Integration.

\section{Ergebnisse}

\section{Chromatographie}

Flüssigkeitschromatogramme von Extrakten eines Serumleerwertes, einer Standard- und einer Patientenprobe zeigt Abbildung 1. Auffällig ist der Metabolitenpeak in der Patientenprobe. Aufgrund des UV-Spektrums handelt es sich vermutlich um den in der Seitenkette carboxylierten Thiopentalmetaboliten; eine weitere Strukturabklärung mittels Massenspektrometrie ist jedoch notwendig.
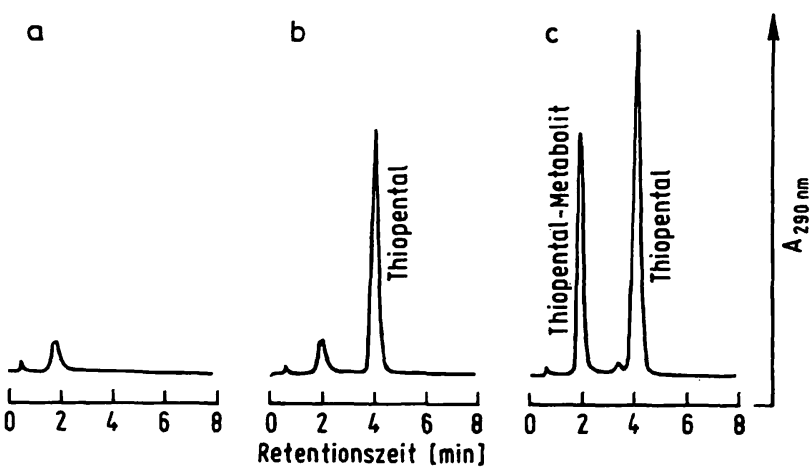

Abb. 1. HPLC-Chromatogramme.

a) Serumleerwert,

b) Serum aufgestockt mit Na-Thiopental (Standard),

c) Patientenserum unter Thiopentaltherapie mit einem Metaboliten von Thiopental (s. Ergebnisse und Diskussion).

\section{Extraktion und Wiederfindung}

Es wurden Extraktionen mit iso-Amylalkohol, $n$ Pentan, $n$-Hexan, $n$-Heptan, Benzol, Butanol, isoButanol, Toluol, Chloroform und Ethylacetat durchgeführt. Hinzu kamen Versuche mit Zusatz von $\mathrm{HCl}$ und $\mathrm{NaCl}$ in verschiedenen Konzentrationen. Die besten Extraktionsausbeuten wurden mit Ethylacetat ohne jeden weiteren Zusatz erzielt. Die Wiederfindung betrug $100 \% \pm 0.7 \%$.

\section{Präzision}

Die Präzision (VK) in der Serie betrug $1,5 \%(\overline{\mathrm{x}}=$ $118 \mu \mathrm{mol} / \mathrm{l}, \mathrm{N}=10)$, die Präzision von Tag zu Tag $3,8 \%(\overline{\mathrm{x}}=75,7 \mu \mathrm{mol} / \mathrm{l}, \mathrm{N}=6)$.

\section{Linearität}

Die Linearität der Methode wurde bis $378 \mu \mathrm{mol} / 1$ Na-Thiopental geprüft, einem Wert, der in der Praxis nie bestimmt wurde. Die Kalibrierkurve verhält sich bis zu diesem Wert linear. "'

Eine Kalibriergerade bis $190 \mu \mathrm{mol} / \mathrm{I} \mathrm{Na}$-Thiopental im Serum zeigt Abbildung 2.

Der Korrelationskoeffizient betrug $r=0,999$, die Regressionsgerade $\mathrm{y}=0,0055 \mathrm{x}+0,243$ (mit $\mathrm{x}$ als Peakfläche).

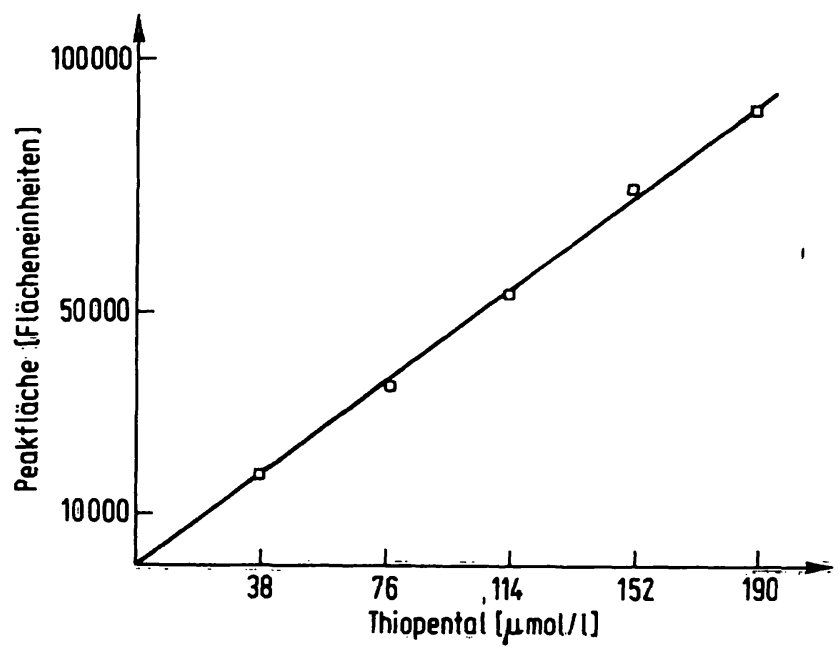

Abb. 2. Standardgerade für die Bestimmung von Thiopentạl im Serum.

\section{Nachweisgrenze}

Bei Einsatz von $100 \mu$ l Serum und einem Injektionsvolumen von $10 \mu \mathrm{l}$ beträgt die für die Praxis relevante Nachweisgrenze $380 \mathrm{nmol} / \mathrm{l}$ (zweifache Höhe des Rauschpegels). Bei Zugrundelegung der doppelten Standardabweichung einer Probe von $380 \mathrm{nmol} / \mathrm{l} \mathrm{er-}$ gibt sich eine Nachweisgrenze von $76 \mathrm{nmol} / \mathrm{l}$.

Unter Verwendung einer größeren Serummenge und eines höheren Injektionsvolumens kann die Nachweisgrenze weiter gesenkt werden, so daß die Methode auch zur Bestimmung der wesentlich geringeren Thiopentalkonzentration innerhalb einer Kurznarkose verwendet werden kann.

\section{Medikamenteneinflüsse}

Folgende in der Intensivtherapie häufig verwendeten Medikamente, in den üblichen Dosierungen verabreicht, wurden auf Interferenzen mit Thiopental bei dessen chromatographischer Bestimmung geprüft: Pethidin, Promethazin, Dihydroergocorninmethansulfonat/Dihydroergocristinmethansulfonat, Flunitrazepam, Pirenzepin, Phentolaminmethansul- 
fonat, Ambroxal, Dexamethason-21-dihydrogenphosphat, Dopamin, Dobutamin, Heparin, Cimetidin, Ranitidin.

Es wurden keine Interferenzen mit dem Thiopentalpeak festgestellt. Ebenso führten Antibiotika aus der Cephalosporin-, Aminoglycosid- oder Penicillinreihe nicht zu Störungen der Thiopentalbestimmung.

Hämolyse bis zu einem Hämoglobingehalt von $20 \mathrm{~g} / 1$ beeinflußt das Meßergebnis nicht.

\section{Therapeutischer Bereich}

Külpmann et al. (6) geben einen vorläufigen therapeutischen Bereich von 124 bis $289 \mu \mathrm{mol} / 1$ an. In unserem Haus wird auf Grund intrakranieller Druckmessungen eine Konzentration im Serum von 76 bis $152 \mu \mathrm{mol} / 1$ als ausreichend erachtet.

\section{Stabilität der Proben}

Untersucht wurde der Einfluß der Probenaufbewahrung auf die nachgewiesene Substanzmenge. Eingefrorene Proben und ebenso solche, die über einen Zeitraum von 10 Tagen bei $4{ }^{\circ} \mathrm{C}$ aufbewahrt wurden, zeigten sich gleichermaßen stabil, während bei Raumtemperatur innerhalb von 10 Tagen die nachzuweisende Menge an Thiopental um etwa 30\% abfiel.

\section{Qualität der Säule}

Mit der verwendeten Säule wurden bereits über 1200 Analysen durchgeführt, ohne daß eine Verschlechterung der Trennleistung festgestellt werden kann. Da diese Säule mit einem gebrochenen, $10 \mu$ Material gefüllt ist, wird ersichtlich, dạ $\not$ unsere $\mathrm{Me}$ thode der Bestimmung von Thiopental an die Säulenqualität keine besongeren Anforderungen stellt.

\section{Diskussion}

Die hier vorgestellte Methode zur Bestimmung von Thiopental im Serum hat sich in unserem Haus zur Uberwachung der Patienten bei hochdosierter Thiopentalanwendung bewährt. Die Methode ist präzise, empfindlich und schnell. Ihr hauptsächlicher Vorzug liegt in der kurzen Probenvorbereitung und der schnellen Chromatographie, so da $B$ eine Bestimmung nur etwa 20 Minuten in Anspruch nimmt. Zudem gestattet derselbe Analysengang die Erfassung des Hauptmetaboliten von Thiopental (Abb. 1). Eine Methode für pharmakokinetische Studien, welche Thiopental, den Hauptmetaboliten und den desulfurierten Metaboliten, Pentobarbital, gleichzeitig er$\mathrm{faßt}$, ist in Bearbeitung. Die klinische Praxis hat gezeigt, daß zur Úberwachung der oben genannten $\mathrm{Pa}$ tienten unter Thiopentaltherapie die Bestimmung von Thiopental allein ausreicht.

Die Verlaufskontrolle der Wirkstoffkonzentration während der Thiopentaltherapie (Abb. 3) zeigt erwartungsgemäß nicht das Bild einer ,reinen“ Pharmakokinetik. Dies rührt her von der fortwährenden und wechselnden Therapie mit mehreren Medikamenten gleichzeitig, insbesonders mit Diuretika und Volumenersatzmitteln, die zu Veränderungen des Verteilungsraumes von Pharmaka führen. Dieses Ergebnis unterstreicht die Notwendigkeit einer engmaschigen Wirkstoffkontrolle: Eine Abschätzung der Thiopentalkonzentration im Serum an Hand der Dosierung ist nicht möglich. Unsere Methode ist geeignet, die für jeden Patienten optimale Dosierung zu finden. Auf Grund der schnellen Analytik kann die Konzentration im Serum sicher im therapeutischen Bereich gehalten werden.

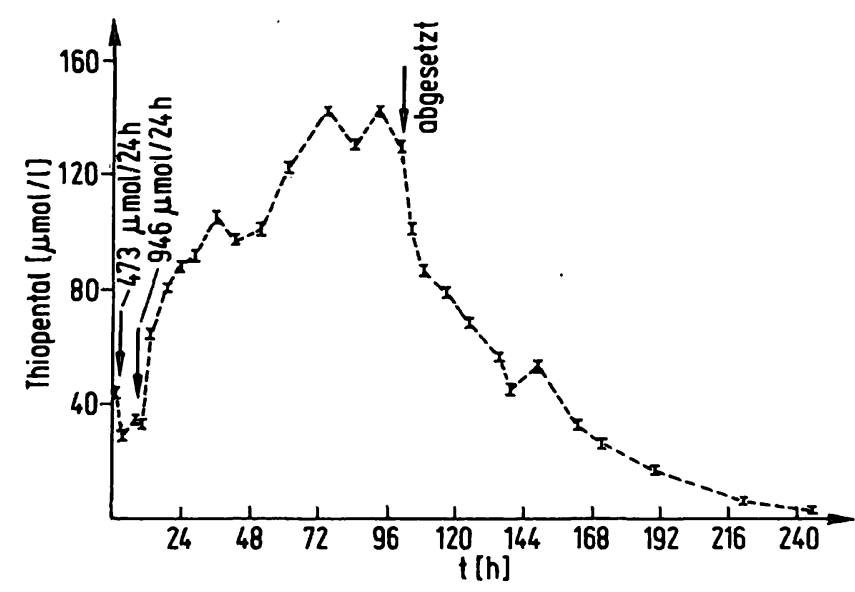

Abb. 3. Thiopentalkonzentration im Serum eines Patienten unter Thiopentaltherapie. Patient E.M. 18 Jahre, 62 kg Körpergewicht, Zustand nach Polytrauma mit Schädelhirnverletzung und generalisiertem Hirnödem. Die senkrechten Pfeile geben die ab diesem Zeitpunkt applizierte Na-Thiopentaldosierung an. Die erste Analyse erfolgte 30 min nach Beginn der Thiopentalapplikation.

\section{Danksagung}

Wir dạnken den Mitarbeitern des Instituts für Anästhesiologic und Intensivmedizin im Hause für die gewissenhaften Blutentnahmen und die gute Zusammenarbeit. 


\section{Literatur}

1. Evelyn, R. (1979) Critical Care Medicine 7, 191.

2. Heuser, D. (1982) Anästhesiologie und Intensivmedizin 23, 315-322

3. Oroszlan, S. I. \& Maengwyn-Davis, G. D. (1960) Am. Pharm. Assoc. Sci. Ed. 49, 507-509.

4. VanHamme, M. J. \& Ghoneim, M. M. (1978) Br. J. Anaesth. 50, 143-145.

5. Jung, D., Mayersohn, M. \& Perrier, D. (1981) Clin. Chem. 27, 113-115.

6. Külpmann, W. R. \& Fitzlaff, R. (1983) J. Clin. Chem. Clin. Biochem. 21, 181-184.

7. Blackman, G. L. \& Jordan, G. J. (1978) J. Chromatogr. 145, 492-495.

8. Masoud, A. N., Scratchley, G. A., Stohs, S. J. \& Wingard, D. W. (1978) J. Liquid Chromatogr. 1, 607-616.

9. Shiu, G. K. \& Nemoto, E. M. (1982) J. Chromatogr. 227, 207-212.

10. Levine, B. \& Blanke, R. (1983) J. Anal. Toxicol. 7, 207208.

Dr. Hartmut Gruhl Institut für Laboratoriumsmedizin

Zentralklinikum

Stenglinstraße

D-8900 Augsburg 